Bulletin of the Section of Logic

Volume 47/4 (2018), pp. 217-232

http://dx.doi.org/10.18778/0138-0680.47.4.01

Tomoaki Kawano

\title{
LABELED SEQUENT CALCULUS FOR ORTHOLOGIC
}

\begin{abstract}
Orthologic (OL) is non-classical logic and has been studied as a part of quantum logic. OL is based on an ortholattice and is also called minimal quantum logic. Sequent calculus is used as a tool for proof in logic and has been examined for several decades. Although there are many studies on sequent calculus for OL, these sequent calculi have some problems. In particular, they do not include implication connective and they are mostly incompatible with the cut-elimination theorem. In this paper, we introduce new labeled sequent calculus called LGOI, and show that this sequent calculus solve the above problems. It is already known that OL is decidable. We prove that decidability is preserved when the implication connective is added to OL.
\end{abstract}

Keywords: quantum logic, sequent calculus, cut-elimination theorem, decidability, Kripke model.

\section{Introduction}

Quantum logic has been studied in the context of orthomodular lattices. An orthomodular lattice is an ortholattice that satisfyies the orthomodular law. Logic based on an ortholattice is referred to as orthologic (OL) or minimal quantum logic, whereas logic based on an orthomodular lattice is referred to as orthomodular logic or quantum logic. In this paper, we discuss OL with an appropriate implication connective. Ortholattices have comparable Kripke semantics, that is, the ortho-model (O-model [1] [7] [8]).

A recurring problem in the study of quantum logic is the implication problem. Negation and conjunction (and disjunction) are the basic connectives in quantum logic because ortholattices and orthomodular lattices can be considered with only these concepts. By contrast, the concept of 
implication is not unique, and there are several implications in quantum logic [1]. However, all these implications are difficult to manage for various reasons, and there is no unified opinion on which implication best suits quantum logic. Among the implications, the most appropriate in quantum logic is the Sasaki arrow $A \rightarrow_{S a} B=\neg(A \wedge \neg(A \wedge B))$ because the meaning and behavior of this implication is useful for both quantum physics and quantum logic [1] [3]. From the viewpoint of physics, $A \rightarrow_{S a} B$ can be translated as " $B$ is true after a state is projected to closed subspace $A$." From the viewpoint of logic, the Sasaki arrow satisfies major requirements for logical implication, for example, modus ponens.

However, regarding OL, the Sasaki arrow is not suitable because it does not satisfy modus ponens in OL. We adopt strict implication $A \rightarrow B$ [1] in place of the Sasaki arrow. Intuitively, $A \rightarrow B$ can be read as "if a state is not orthogonal to the current state, and if $A$ is true in that state, then $B$ is also true in that state." Strict implication satisfies major requirements for logical implication, including modus ponens. Strict implication cannot be defined in terms of other logical connectives [1], which means that the expressive power of OL increases as a result of the addition of strict implication. For these reasons, strict implication can be considered as the most appropriate implication in OL. However, it has the disadvantage of lacking a deduction system. In this paper, we overcome this lack, as explained below.

Some deduction systems in OL are types of sequent calculi. However, almost all sequent calculi for OL are incompatible with the cut-elimination theorem. For example, the sequent calculus GO [7] has a cut rule, but we cannot remove it. Conversely, GMQL [8] [9] does not have a (full) cut rule. If a cut rule is added to GMQL, then it collapses to classical logic. Sequent calculi for OL that satisfy the cut-elimination theorem were proposed in [2]. However, it is difficult to add strict implication to this calculi. In [2], Faggian and Sambin considered $\neg p(p \rightarrow \perp$ in this paper) as an atomic formula. Because $p \rightarrow \perp$ is a special case of $A \rightarrow B$, when we attempt to add new rules for strict implication into this system, we have to consider $p \rightarrow \perp$ and $A \rightarrow B$ separately. Therefore, even if we succeed in making rules, the rules may be more complicated than necessary.

To overcome these problems, we need an advanced sequent calculus. We adopt labeled sequent calculus, called LGOI in this paper, because this type of calculus is compatible with implication. A labeled sequent includes not only formulas but also relations based on a Kripke frame. This type 
of sequent calculus has been used to prove the cut-elimination theorem [4] [5]. Details and the development of labeled sequent calculi are summarized in [6]. In this paper, we construct LGOI and prove the soundness and cut-free completeness theorem. The cut-elimination theorem is a corollary of these theorems.

We also prove that LGOI is decidable. It is already known that the validity problem for OL is decidable; that is, there exists an effective procedure to decide whether a formula is valid in O-models. In this paper, we prove that the validity problem for OL with strict implication is also decidable. Although the decidability of the validity problem for OL with strict implication is derived from the decidability of LGOI using the completeness theorem, we first prove the former theorem for convenience.

In Section 2, we discuss the basics of OL. In Section 3, we discuss LGOI and the soundness theorem for it. In Section 4, we discuss the completeness theorem for LGOI and the decidability of orthologic with strict implication.

\section{Basics}

We use a language that has a denumerable infinite set of propositional variables, the propositional constant $\perp$, and the binary connectives $\wedge$ and $\rightarrow$. We use $p, q, r, \ldots$ to denote propositional variables and $A, B, C, \ldots$ to denote formulas. Formulas are constructed in the usual way. We use $\neg A$ as an abbreviation of $A \rightarrow \perp$. We take a countably infinite set to be the set of labels, and use $a, b, c, \ldots$ to denote the labels. We define the complexity of formula $A$ as the number of connectives that appear in $A$. For example, the complexity of $p \wedge(\neg q \wedge r)$ is 3 .

An $O$-frame is a pair $(X, \perp)$, where $X$ is a non-empty set and $\perp$ is a binary relation on $X$ that is irreflexive and symmetric. We abuse the symbol $\perp$ in two ways: as a relation and formula. For $x \in X$ and $Y \subseteq X$, we write $x \perp Y$ if for all $y \in Y, x \perp y$. Given $Y \subseteq X$, we define the set $Y^{\perp}$ $=\{x \in X \mid x \perp Y\}$. We say that $Y$ is $\perp$-closed if $Y^{\perp \perp}=Y$. We write $x \not \perp y$ if not $x \perp y$. As $\perp$ is an irreflexive symmetric relation, we can regard $\not \perp$ as a reflexive symmetric relation on $X$.

Lemma 2.1 (Fundamental properties of a $\perp$-closed set). For any set $Y \subseteq$ $X$, we have $Y \subseteq Y^{\perp \perp}$. For any set $Y \subseteq X$, the set $Y^{\perp}$ is $\perp$-closed. If $Y \subseteq X$ and $Z \subseteq X$ are $\perp$-closed, then $Y \cap Z$ is $\perp$-closed. 
Proof: First, we prove the first claim. For all $x \in Y^{\perp}$ and for all $y \in Y$, $y \perp x$ because of the definition of $Y^{\perp}$ and symmetry of $\perp$. Therefore, $Y \subseteq$ $\left\{z \in X \mid z \perp Y^{\perp}\right\}=Y^{\perp \perp}$.

Next, we prove the second claim. Suppose that $x \in Y^{\perp \perp \perp}$. From $Y \subseteq Y^{\perp \perp}, x \perp Y$. Therefore, from $x \in Y^{\perp}$ and $Y^{\perp} \subseteq Y^{\perp \perp \perp}, Y^{\perp \perp \perp}=Y^{\perp}$.

Next, we prove the third claim. For the sake of contradiction, suppose that there exists $x \in(Y \cap Z)^{\perp \perp}$ such that $x \notin Y \cap Z$. Because $Y \cap Z \subseteq Y$, for all $y \in Y^{\perp}, y \perp(Y \cap Z)$. Therefore, $Y^{\perp} \subseteq(Y \cap Z)^{\perp}$. For the same reason, $Z^{\perp} \subseteq(Y \cap Z)^{\perp}$. From $x \notin Y \cap Z, x \notin \bar{Y}$ or $x \notin Z$. Suppose $x \notin Y$. From $Y=Y^{\perp \perp}$, there exists $y \in Y^{\perp}$ such that $x \not \perp y$. However, from $y \in Y^{\perp} \subseteq(Y \cap Z)^{\perp}$ and $x \in(Y \cap Z)^{\perp \perp}, x \perp y$. This is a contradiction. Suppose $x \notin Z$. Then, the same proof that have been described can be applied.

An $O$-model is a triple $(X, \perp, V)$, where $(X, \perp)$ is an O-frame, and $V$ is a function that assigns each propositional variable $p$ to a $\perp$-closed subset of $X$. We define set $\|A\|$ by induction on the composition of $A$ as follows:

$$
\begin{aligned}
& \|p\|=V(p), \\
& \|A \wedge B\|=\|A\| \cap\|B\|, \\
& \|A \rightarrow B\|=\{x \in X \mid \text { for all } y \in X, \text { if } x \not \perp y \text { and } y \in\|A\|, \text { then } y \in\|B\|\}, \\
& \|\perp\|=\emptyset \text {. }
\end{aligned}
$$

We say that a formula $A$ is true at $x \in X$ if $x \in\|A\|$, and write $x \models A$. We say that a formula $A$ is false at $x \in X$ if $A$ is not true at $x$.

It is easy to check that $\|A \rightarrow \perp\|$ is equal to $\|A\|^{\perp}(=\|\neg A\|$ in [7] [8]). TheOrem 2.2. For all $\|A\|$, the set $\|A\|$ is $\perp$-closed.

Proof: We prove this by induction on the construction of formula $A$.

For atomic formulas, it is obvious from the definition.

Suppose $A=B \wedge C$. From the inductive hypothesis, $\|B\|$ and $\|C\|$ are $\perp$-closed. Then, from Lemma 2.1, $\|B \wedge C\|=\|B\| \cap\|C\|$ is $\perp$-closed.

Suppose $A=B \rightarrow C$. Since $\|B \rightarrow C\|$ is equal to $(\|B\| \cap(X \backslash\|C\|))^{\perp}$, from Lemma 2.1, $\|B \rightarrow C\|$ is $\perp$-closed.

A labeled formula is defined as $a: A$, where $a$ is a label and $A$ is a formula. We use $\Gamma, \Delta, \Sigma, \ldots$ to denote sets of labeled formulas. We say that label $a$ appears in $\Gamma$ if $a: A$ is included in $\Gamma$ for some $A$. We define a relational expression as $a \not \perp b$ ( $a$ and $b$ are any labels).

A labeled sequent is defined as $\{R\} \Gamma \Rightarrow \Delta$, where $\{R\}$ is a set of relational expressions, all $\{R\}, \Gamma$, and $\Delta$ are finite sets. We say that label 
$a$ appears in set $\{R\}$ if $a \not \perp b$ or $b \not \perp a$ is included in $\{R\}$ for some $b$. We use $\{R\}_{L}$ to denote the set of all labels that appear in $\{R\}$. We use $(\{R\} \Gamma \Rightarrow \Delta)_{L}$ to denote the set of all labels that appear in $\{R\} \Gamma \Rightarrow \Delta$. We define $\{R, a \not \perp b\}=\{R\} \cup\{a \not \perp b\}$.

An embedding of a labeled sequent $\{R\} \Gamma \Rightarrow \Delta$ in an O-model $(X, \perp, V)$ is a function $E$ from $(\{R\} \Gamma \Rightarrow \Delta)_{L}$ to $X$ that satisfies the following condition:

If $a \not \perp b \in\{R\}, E(a)=x$, and $E(b)=y$, then $x \not \perp y$.

We say that a labeled sequent $\{R\} \Gamma \Rightarrow \Delta$ is true in $(X, \perp, V)$ under an embedding $E$ if, whenever $A$ is true at $E(a)$ for all $a: A \in \Gamma, B$ is true at $E(b)$ for some $b: B \in \Delta$. We say that a labeled sequent $\{R\} \Gamma \Rightarrow \Delta$ is false in $(X, \perp, V)$ under an embedding $E$ if $\{R\} \Gamma \Rightarrow \Delta$ is not true in $(X, \perp, V)$ under $E$. We say that a labeled sequent $\{R\} \Gamma \Rightarrow \Delta$ is valid in O-models if $\{R\} \Gamma \Rightarrow \Delta$ is true for all O-models and for all embeddings $E$.

An infinite labeled sequent is defined as $\{R\} \Gamma \Rightarrow \Delta$, where $\{R\}, \Gamma$, or $\Delta$ is an infinite set, and the other conditions are the same as those for labeled sequents. We say that labeled sequent $\left\{R^{\prime}\right\} \Gamma^{\prime} \Rightarrow \Delta^{\prime}$ is a subsequent of $\{R\} \Gamma \Rightarrow \Delta$ if $\{R\}^{\prime} \subseteq\{R\}, \Gamma^{\prime} \subseteq \Gamma$, and $\Delta^{\prime} \subseteq \Delta$.

\section{Labeled sequent calculus LGOI}

In this section, we discuss LGOI for OL with strict implication, and prove the soundness theorem. LGOI is defined as follows, where the sets $\{R\}$, $\Gamma$, and $\Delta$ are finite:

Axiom:

$$
\{R\} a: A \Rightarrow a: A \quad\{R\} a: \perp \Rightarrow
$$

Rules:

$$
\begin{gathered}
\frac{\{R\} \Gamma \Rightarrow \Delta, a: A \quad\{R\} a: A, \Gamma \Rightarrow \Delta}{\{R\} \Gamma \Rightarrow \Delta} \text { (cut) } \\
\frac{\{R\} \Gamma \Rightarrow \Delta}{\{R\} a: A, \Gamma \Rightarrow \Delta}(\mathrm{wL}) \quad \frac{\{R\} \Gamma \Rightarrow \Delta}{\{R\} \Gamma \Rightarrow \Delta, a: A}(\mathrm{wR}) \\
\frac{\{R\} a: A, a: B, \Gamma \Rightarrow \Delta}{\{R\} a: A \wedge B, \Gamma \Rightarrow \Delta}(\wedge \mathrm{L}) \frac{\{R\} \Gamma \Rightarrow \Delta, a: A \quad\{R\} \Gamma \Rightarrow \Delta, a: B}{\{R\} \Gamma \Rightarrow \Delta, a: A \wedge B}(\wedge \mathrm{R})
\end{gathered}
$$




$$
\begin{aligned}
& \frac{\{R, a \not \perp b\} \Gamma \Rightarrow \Delta, b: A \quad\{R, a \not \perp b\} b: B, \Gamma \Rightarrow \Delta}{\{R, a \not \perp b\} a: A \rightarrow B, \Gamma \Rightarrow \Delta}(\rightarrow \mathrm{L}) \\
& \frac{\{R, a \not \perp b\} b: A, \Gamma \Rightarrow \Delta, b: B}{\{R\} \Gamma \Rightarrow \Delta, a: A \rightarrow B}(\rightarrow \mathrm{R}) \\
& \frac{\{R, a \not \perp b\} b: \neg p, \Gamma \Rightarrow \Delta}{\{R\} \Gamma \Rightarrow \Delta, a: p}(\neg)
\end{aligned}
$$

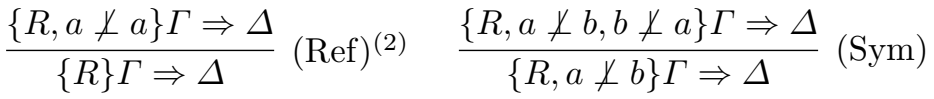

$$
\begin{aligned}
& \frac{\{R\} \Gamma \Rightarrow \Delta}{\{R, a \not \perp b\} \Gamma \Rightarrow \Delta}(\text { wRe })
\end{aligned}
$$

(1) In rules $(\rightarrow R)$ and $(\neg), b$ must not appear in $\{R\}, \Gamma$, and $\Delta$.

(2) In rules (Ref), a must appear in $\{R\}, \Gamma$, or $\Delta$.

An example of the derivations in LGOI is provided below. We can confirm that sequent $A, B \Rightarrow \neg(C \wedge \neg(A \wedge B))$, which is not provable without rule (cut) in [7], is now provable without (cut) in LGOI.

$$
\begin{aligned}
& \frac{\{a \not \supset b, b \not \perp a\} a: A \Rightarrow a: A}{\{a \not \perp b, b \not \perp a\} a: A, a: B, b: C \Rightarrow a: A, b: \perp}(\mathrm{wL}) \times 2,(\mathrm{wR}) \times 1
\end{aligned}
$$

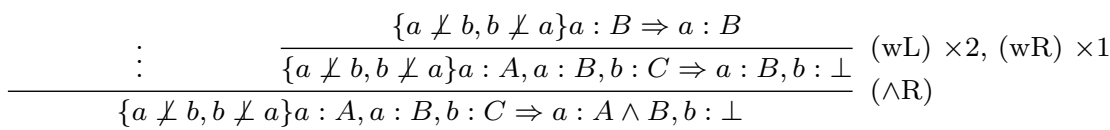

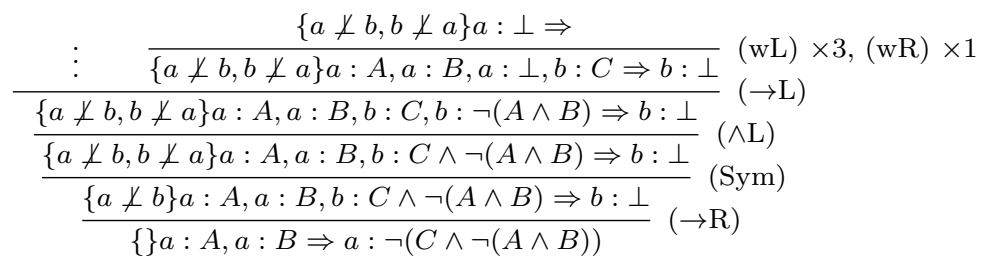

Before the proofs of the soundness theorem, we make remarks on rule $(\neg)$. Unfortunately, rule $(\neg)$ does not satisfy the subformula property, where the property means that each labeled formula $a: A$ in the premise of a rule is a subformula of a labeled formula in the conclusion of the rule. 
Without this rule, it is difficult to make complete labeled sequent calculus (using $\not \perp$ as relations) of OL that satisfies the cut-elimination theorem for reasons stated below.

If $(\neg)$ is removed from LGOI- $\{($ cut $)\}$, we cannot prove $\{R\} a: \neg \neg(p \wedge$ $q) \Rightarrow a: p$, which is provable in LGOI- $\{($ cut $)\}$. From the shape of the formulas in $\{R\} a: \neg \neg(p \wedge q) \Rightarrow a: p$ and the subformula property of LGOI- $\{($ cut $),(\neg)\}$, if $\{R\} a: \neg \neg(p \wedge q) \Rightarrow a: p$ is provable, then the axioms of the proof must be $\left\{R^{\prime}\right\} a: p \Rightarrow a: p$. However, we can check that regardless of how we reverse the deduction from $\{R\} a: \neg \neg(p \wedge q) \Rightarrow a: p$, we cannot reach $\left\{R^{\prime}\right\} a: p \Rightarrow a: p$. Therefore, this sequent is not provable in LGOI- $\{$ (cut), $(\neg)\}$. If we can use $(\neg)$, then $\{R\} a: \neg \neg(p \wedge q) \Rightarrow a: p$ is provable, as shown below.

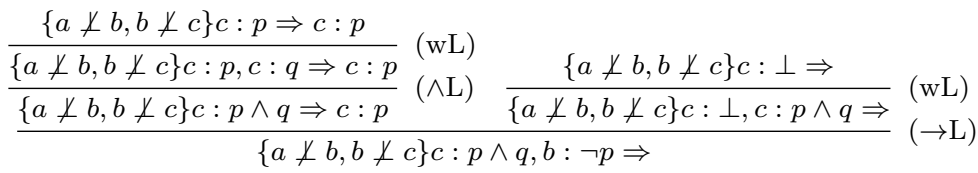

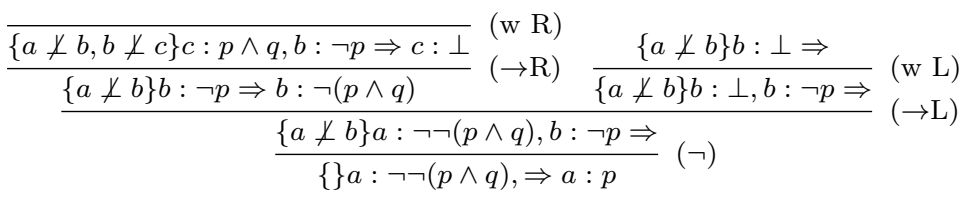

Furthermore, $\{R\} a: \neg \neg(p \wedge q) \Rightarrow a: p$ has no proof in LGOI- $\{($ cut $)\}$ that all deductions are subformulaic. Because we have to use rule $(\neg)$ to prove this sequent, $\neg p$ must appear somewhere in the proof. However, as $\neg p$ is not a subformula of $\neg \neg(p \wedge q)$ or $p$, there exist some deductions that are not subformulaic in the proof of $\{R\} a: \neg \neg(p \wedge q) \Rightarrow a: p$.

In the proof of the cut-free completeness theorem, we need rule $(\neg)$ to establish the falsity of $p$ in a canonical model, as we show in the proof of Lemma 4.1. The falsity of $p$ depends on not only $p$ but also $\neg p$ because of the nature of a $\perp$-closed set; that is, in an O-model $(X, \perp, V), p$ is false at $x \in X$ if and only if there exists $y \in X$ such that $y \in\|\neg p\|$ and $x \not \perp y$.

Moreover, we can show that rule $(\neg)$ corresponds to condition " $\perp$ closed" of O-models. We define a pseudo-O-model as $\left(X, \perp, V^{\prime}\right)$, where the definitions of $X$ and $\perp$ are the same as those of an O-model, and $V^{\prime}$ is a 
function that assigns each propositional variable $p$ to a subset of $X$; that is, $V^{\prime}(p)$ does not need to be a $\perp$-closed set. Then, LGOI- $\{($ cut $),(\neg)\}$ is sound and complete with respect to pseudo-O-models (Theorem 4.4).

To conclude, rule $(\neg)$ is necessary to prove the cut-free completeness theorem; however, it destroys the subformula property. Thus, a natural question arises: Does a labeled sequent calculus for $O L$ that satisfies the subformula property exist? This is an interesting open problem.

Now, we prove the soundness theorem.

TheOREM 3.1 (Soundness theorem for LGOI). If $\{R\} \Gamma \Rightarrow \Delta$ is provable in LGOI, then $\{R\} \Gamma \Rightarrow \Delta$ is valid in O-models.

Proof: We prove this by induction on the construction of the proof of sequent $\{R\} \Gamma \Rightarrow \Delta$. We only prove the cases for which the last rule used in the proof is $(\rightarrow \mathrm{L}),(\rightarrow \mathrm{R})$, (Ref), or (Sym). The proofs for the other cases are similar. The proof for $(\neg)$ is the similar to that for $(\rightarrow \mathrm{R})$ because $\|p\|=\|\neg \neg p\|=\|\neg p \rightarrow \perp\|$.

First, we show the case in which the last rule is $(\rightarrow \mathrm{L})$. Suppose that $\{R, a \not \perp b\} a: A \rightarrow B, \Gamma \Rightarrow \Delta$ is false in an O-model $(X, \perp, V)$ under embedding $E$. Then, $A$ is false at $E(b)$ or $B$ is true at $E(b)$. Therefore, $\{R, a \not \perp b\} \Gamma \Rightarrow \Delta, b: A$ is false under $E$ or $\{R, a \not \perp b\} b: B, \Gamma \Rightarrow \Delta$ is false under $E$.

Next, we show the case in which the last rule is $(\rightarrow \mathrm{R})$. For the sake of contradiction, suppose that $\{R, a \not \perp b\} b: A, \Gamma \Rightarrow \Delta, b: B$ is valid and that $\{R\} \Gamma \Rightarrow \Delta, a: A \rightarrow B$ is false in $(X, \perp, V)$ under $E$. Then there exists $x \in X$ such that $E(a) \not \perp x$ and $A$ is true at $x$ but $B$ is not true at $x$. If we define $E^{\prime}$ as $E \cup\{(b, x)\}$, then $\{R, a \not \perp b\} b: A, \Gamma \Rightarrow \Delta, b: B$ is false in $(X, \perp, V)$ under $E^{\prime}$. This is a contradiction. Therefore, $\{R\} \Gamma \Rightarrow \Delta, a$ : $A \rightarrow B$ is valid.

Next, we show the case in which the last rule is (Ref). Because relation $\not \perp$ in an O-model is reflexive and $a$ appears in $\{R\} \Gamma \Rightarrow \Delta,\{R, a \not \perp a\} \Gamma \Rightarrow$ $\Delta$ and $\{R\} \Gamma \Rightarrow \Delta$ have the same embeddings. Therefore, if $\{R, a \not \perp a\} \Gamma \Rightarrow$ $\Delta$ is valid, then $\{R\} \Gamma \Rightarrow \Delta$ is also valid.

Next, we show the case in which the last rule is (Sym). Because the relation $\not \perp$ in an O-model is symmetric, $\{R, a \not \perp b, b \not \perp a\} \Gamma \Rightarrow \Delta$ and $\{R, a \not \perp b\} \Gamma \Rightarrow \Delta$ have the same embeddings. Therefore, if $\{R, a \not \perp b, b \not \perp$ $a\} \Gamma \Rightarrow \Delta$ is valid, then $\{R, a \not \perp b\} \Gamma \Rightarrow \Delta$ is also valid. 


\section{Completeness theorem for LGOI}

In this section, we prove the cut-free completeness theorem for LGOI, and also prove that orthologic with strict implication is decidable.

For the cut-free completeness theorem, we prove the contrapositive; that is, if the labeled sequent $\{R\} \Gamma \Rightarrow \Delta$ is not provable in LGOI- $\{$ (cut) $\}$, then there exists a model $(X, \perp, V)$ and embedding $E$ of $\{R\} \Gamma \Rightarrow \Delta$ to $(X, \perp, V)$ such that $\{R\} \Gamma \Rightarrow \Delta$ is false in $(X, \perp, V)$ under $E$. We construct a canonical model $\left(X_{c}, \perp_{c}, V_{c}\right)$ from an unprovable sequent $\{R\} \Gamma \Rightarrow \Delta$ by creating new labels and relations while preserving unprovability. We denote these changes in the sequent by $\{R\}_{0} \Gamma_{0} \Rightarrow \Delta_{0}(=\{R\} \Gamma \Rightarrow \Delta),\{R\}_{1} \Gamma_{1} \Rightarrow$ $\Delta_{1}, \ldots,\{R\}_{i} \Gamma_{i} \Rightarrow \Delta_{i},\{R\}_{i+1} \Gamma_{i+1} \Rightarrow \Delta_{i+1}, \ldots$

Suppose that $\{R\} \Gamma \Rightarrow \Delta$ is not provable in LGOI- $\{($ cut $)\}$.

Step 1: We make a new sequent by applying the following procedure until the sequent does not change.

- If $a:(A \wedge B) \in \Gamma_{i}$, then we define $\Gamma_{i+1}=\Gamma_{i} \cup\{a: A, a: B\}$ $\Delta_{i+1}=\Delta_{i}$ and $\{R\}_{i+1}=\{R\}_{i}$. Sequent $\{R\}_{i+1} \Gamma_{i+1} \Rightarrow \Delta_{i+1}$ is not provable because of rule $(\wedge \mathrm{L})$.

- If $a:(A \wedge B) \in \Delta_{i}$, then at least one of $\{R\}_{i} \Gamma_{i} \Rightarrow \Delta_{i}, a: A$ and $\{R\}_{i} \Gamma_{i} \Rightarrow \Delta_{i}, a: B$ is not provable because of rule $(\wedge \mathrm{R})$. We define $\Delta_{i+1}=\Delta_{i} \cup\{a: A\}$ or $\Delta_{i} \cup\{a: B\}$, which preserves unprovability. In either case, we define $\Gamma_{i+1}=\Gamma_{i}$ and $\{R\}_{i+1}=$ $\{R\}_{i}$.

- If $a:(A \rightarrow B) \in \Gamma_{i}$ and $a \not \perp b \in\{R\}$, then at least one of $\{R\}_{i} \Gamma_{i} \Rightarrow \Delta_{i}, b: A$ and $\{R\}_{i} \Gamma_{i}, b: B \Rightarrow \Delta_{i}$ is not provable because of rule $(\rightarrow \mathrm{L})$. We define $\Gamma_{i+1}=\Gamma_{i} \cup\{b: B \mid a \not \perp b \in\{R\}$ and $\{R\}_{i} \Gamma_{i}, b: B \Rightarrow \Delta_{i}$ is not provable $\}$ and $\Delta_{i+1}=\Delta_{i} \cup\{b$ : $A \mid a \not \perp b \in\{R\}$ and $\{R\}_{i} \Gamma_{i} \Rightarrow \Delta_{i}, b: A$ is not provable $\}$. In each case, we define $\{R\}_{i+1}=\{R\}_{i}$.

- If $a:(A \rightarrow B) \in \Delta_{i}$ and we have not executed this procedure on this formula, then we take label $b$ that does not appear in $\{R\}_{i} \Gamma_{i} \Rightarrow \Delta_{i}$ and define $\Gamma_{i+1}=\Gamma_{i} \cup\{b: A\}, \Delta_{i+1}=\Delta_{i} \cup\{b:$ $B\}$ and $\{R\}_{i+1}=\{R, a \not \perp b\}_{i}$. The sequent $\{R\}_{i+1} \Gamma_{i+1} \Rightarrow$ $\Delta_{i+1}$ is not provable because of rule $(\rightarrow \mathrm{R})$.

Note that we can add only one label $b$ for $a:(A \rightarrow B) \in \Delta_{i}$ because of the condition. As all of these procedures decrease the complexity 
of the formulas, this procedure ends at some point. We then go to Step 2.

Step 2: We define a new sequent by applying the following procedure until the sequent does not change.

- If $a: p \in \Delta_{i}$ and we have not executed this procedure on this formula, then we take label $b$ that does not appear in $\{R\}_{i} \Gamma_{i} \Rightarrow \Delta_{i}$ and define $\Gamma_{i+1}=\Gamma_{i} \cup\{b: \neg p\}, \Delta_{i+1}=\Delta_{i}$ and $\{R\}_{i+1}=\{R, a \not \perp b\}_{i}$. Sequent $\{R\}_{i+1} \Gamma_{i+1} \Rightarrow \Delta_{i+1}$ is not provable because of rule $(\neg)$.

- If label $a$ appears in $\{R\}_{i} \Gamma_{i} \Rightarrow \Delta_{i}$ and if $a \not \perp a$ is not included in $\{R\}_{i}$, then we define $\{R\}_{i+1}=\{R, a \not \perp a\}_{i}, \Gamma_{i+1}=\Gamma_{i}$ and $\Delta_{i+1}=\Delta_{i}$. Sequent $\{R\}_{i+1} \Gamma_{i+1} \Rightarrow \Delta_{i+1}$ is not provable because of rule (Ref).

- If $a \not \perp b$ is included in $\{R\}_{i}$ and if $b \not \perp a$ is not included in $\{R\}_{i}$, then we define $\{R\}_{i+1}=\{R, b \not \subset a\}_{i}, \Gamma_{i+1}=\Gamma_{i}$ and $\Delta_{i+1}=\Delta_{i}$. Sequent $\{R\}_{i+1} \Gamma_{i+1} \Rightarrow \Delta_{i+1}$ is not provable because of rule (Sym).

This step ends at some point because the number of labels and propositional variables that appear in $\{R\}_{i} \Gamma_{i} \Rightarrow \Delta_{i}$ are finite. We now return to Step 1 because new labels and relations are created in Step 2.

We say that an infinite labeled sequent is not provable if all finite subsequents are not provable. We define the infinite labeled sequent $\{R\}_{c} \Gamma_{c} \Rightarrow$ $\Delta_{c}$ as $\{R\}_{c}=\bigcup_{i=0}^{\infty}\{R\}_{i}, \Gamma_{c}=\bigcup_{i=0}^{\infty} \Gamma_{i}$ and $\Delta_{c}=\bigcup_{i=0}^{\infty} \Delta_{i}$; that is, this sequent is the limit of Step $1 \rightarrow$ Step $2 \rightarrow$ Step $1 \rightarrow$ Step $2 \rightarrow \ldots$. The nature of the above procedure confirms that this infinite labeled sequent is not provable. That is, for all finite subsequents $\{R\}^{\prime} \Gamma^{\prime} \Rightarrow \Delta^{\prime}$ of $\{R\}_{c} \Gamma_{c} \Rightarrow \Delta_{c}$, there exists $i$ such that $\{R\}^{\prime} \subseteq\{R\}_{i}, \Gamma^{\prime} \subseteq \Gamma_{i}$, and $\Delta^{\prime} \subseteq \Delta_{i}$. If $\{R\}^{\prime} \Gamma^{\prime} \Rightarrow \Delta^{\prime}$ is provable, then $\{R\}_{i} \Gamma_{i} \Rightarrow \Delta_{i}$ is provable because of (wL), (wR), and (wRe).

We define canonical model $\left(X_{c}, \perp_{c}, V_{c}\right)$ for $\{R\} \Gamma \Rightarrow \Delta$ as follows:

$$
\begin{aligned}
& X_{c}=\left(\{R\}_{c}\right)_{L}, \\
& \perp_{c}=\left\{(a, b) \mid a \not \perp b \notin\{R\}_{c}\right\}, \\
& V_{c}(p)=\left\{a \mid a: p \in \Gamma_{c}\right\}^{\perp \perp} .
\end{aligned}
$$

From Lemma 2.1, set $\|p\|$ is $\perp$-closed for all $p$ in $\left(X_{c}, \perp_{c}, V_{c}\right)$. From Step $2, \perp_{c}$ is irreflexive and symmetric. Therefore, $\left(X_{c}, \perp_{c}, V_{c}\right)$ is actually an O-model. 
We cannot use the natural definition $\left\{a \mid a: p \in \Gamma_{c}\right\}$ for $V_{c}(p)$ because $\left\{a \mid a: p \in \Gamma_{c}\right\}$ may not be a $\perp$-closed set. Because $Y \subseteq Y^{\perp \perp}$, there may be exist $a \in X_{c}$ such that $a \in V_{c}(p)$ and $a: p \notin \Gamma_{c}$. This feature does not cause problems, as shown in Lemma 4.1; that is, there is no $a \in X_{c}$ such that $a \in V_{c}(p)$ and $a: p \in \Delta_{c}$.

We define embedding $E_{c}$ of $\{R\} \Gamma \Rightarrow \Delta$ to $\left(X_{c}, \perp_{c}, V_{c}\right)$ as $E_{c}(a)=a$. Lemma 4.1. If $a: A \in \Gamma_{c}$, then $A$ is true at $a \in X_{c}$. If $a: A \in \Delta_{c}$, then $A$ is false at $a \in X_{c}$. Therefore, $\{R\} \Gamma \Rightarrow \Delta$ is false in $\left(X_{c}, \perp_{c}, V_{c}\right)$ under $E_{c}$. Proof: We prove this lemma by induction on the construction of the labeled formulas in $\Gamma_{c}$ and $\Delta_{c}$. Suppose that $a: p \in \Gamma_{c}$. Then, from the definition of $V_{c}$ and Lemma 2.1, we have $a \in\|p\|$. Suppose that $a: p \in \Delta_{c}$. From Step 2, there exists label $b$ that satisfies $b: \neg p \in \Gamma_{c}$ and $a \not \perp b \in\{R\}_{c}$. If there exists some label $c$ that satisfies $b \not \perp c \in\{R\}_{c}$ and $c: p \in \Gamma_{c}$, then $\{R\}_{i} \Gamma_{i} \Rightarrow \Delta_{i}$ is provable for some $i$ because $\{R, b \not \perp c\} \Gamma \cup\{b: \neg p, c: p\} \Rightarrow$ $\Delta$ is provable. This is a contradiction. Therefore, if $b: \neg p \in \Gamma_{c}$, then $b \in\left\{a \mid a: p \in \Gamma_{c}\right\}^{\perp}=\left\{a \mid a: p \in \Gamma_{c}\right\}^{\perp \perp \perp}=\|\neg p\|$ by the definition of $V_{c}$ and Lemma 2.1. Therefore, $a \notin\|p\|$.

Suppose that $a: A \wedge B \in \Gamma_{c}$. From Step 1, $a: A \in \Gamma_{c}$ and $a: B \in \Gamma_{c}$. From the induction hypothesis, $A$ and $B$ are true at $a \in X_{c}$. Therefore, $A \wedge B$ is true at $a \in X_{c}$.

Suppose that $a: A \wedge B \in \Delta_{c}$. From Step 1, $a: A \in \Delta_{c}$ or $a: B \in \Delta_{c}$. From the induction hypothesis, $A$ or $B$ is false at $a \in X_{c}$. Therefore, $A \wedge B$ is false at $a \in X_{c}$.

Suppose that $a: A \rightarrow B \in \Gamma_{c}$. From Step 1, $b: A \in \Delta_{c}$ or $b: B \in \Gamma_{c}$ for all labels $b$ such that $a \not \perp b \in\{R\}_{c}$. From the induction hypothesis, $A$ is false at $b \in X_{c}$ or $B$ is true at $b \in X_{c}$. Therefore, $A \rightarrow B$ is true at $a \in X_{c}$.

Suppose that $a: A \rightarrow B \in \Delta_{c}$. From Step 1, there exists a label $b$ such that $a \not \perp b \in\{R\}_{c}, b: A \in \Gamma_{c}$ and $b: B \in \Delta_{c}$. From the induction hypothesis, $A$ is true at $b \in X_{c}$ and $B$ is false at $b \in X_{c}$. Therefore, $A \rightarrow B$ is false at $a \in X_{c}$.

THEOREM 4.2 (Cut-free completeness theorem for LGOI). If $\{R\} \Gamma \Rightarrow \Delta$ is valid in $O$-models, then $\{R\} \Gamma \Rightarrow \Delta$ is provable in LGOI without rule (cut).

Proof: From Lemma 4.1, if $\{R\} \Gamma \Rightarrow \Delta$ is not provable in LGOI- $\{($ cut $)\}$, then there exists an O-model $(X, \perp, V)$ and embedding $E$ such that $\{R\} \Gamma \Rightarrow \Delta$ is false in $(X, \perp, V)$ under $E$. 
Theorem 4.3 (Cut-elimination theorem for LGOI). If $\{R\} \Gamma \Rightarrow \Delta$ is provable in LGOI, then there exists a proof of $\{R\} \Gamma \Rightarrow \Delta$ that does not include rule (cut).

Proof: Corollary of Theorem 3.1 and Theorem 4.2.

TheOREM 4.4 (Soundness and completeness theorems for LGOI- $\{(\mathrm{cut})$, $(\neg)\})$. A labeled sequent $\{R\} \Gamma \Rightarrow \Delta$ is provable in LGOI- $\{($ cut $),(\neg)\}$ if and only if $\{R\} \Gamma \Rightarrow \Delta$ is valid in pseudo-O-models.

Proof: For the soundness theorem, because we did not use the notion of a $\perp$-closed set in the proof of Theorem 3.1 except for rule $(\neg)$, we can use the same proof.

For the completeness theorem, we can make a canonical model for $\{R\} \Gamma \Rightarrow \Delta$ by applying all procedures in Step 1 and procedures for (Ref) and (Sym) in Step 2. We use $\left\{a \mid a: p \in \Gamma_{c}\right\}$ for $V_{c}^{\prime}(p)$. Then, we can use almost the same proof as that of Lemma 4.1; however, it is much easier because we do not need to be concerned about $\perp$-closedness of sets.

We can now prove that the validity problem for OL with strict implication is decidable using the completeness theorem. We can check the provability of labeled sequent \{\}$\Rightarrow a: A$ by checking whether a canonical model for \{\}$\Rightarrow a: A$ exists. We can construct a canonical model that is essentially equivalent to $\left(X_{c}, \perp_{c}, V_{c}\right)$ in a finite number of steps. We execute the same procedure to construct $\{R\}_{i} \Gamma_{i} \Rightarrow \Delta_{i}$ but we stop the procedure at some $i$.

Suppose that label $b$ is included in $\{R\}_{i}$ in the procedure for constructing a canonical model for \{\}$\Rightarrow a: A$. We say that the degree of label $b$ for $a$ is $n(\geq 2)$ in $\{R\}_{i}$ if there exist labels $c_{1}, c_{2}, \ldots, c_{n-1}$ such that $a \not \perp c_{1}, c_{1} \not \perp c_{2}, \ldots, c_{n-1} \not \perp b$ are included in $\{R\}_{i}$ and $a, b, c_{1}, c_{2}, \ldots, c_{n-1}$ are distinct labels. We define the degree of $a$ as 0 , and if $a \not \perp b \in\{R\}_{i}$, then we define the degree of $b(\neq a)$ as 1 . The degree of a label is uniquely determined because we can confirm that there is no confluence (or loop) such as $d_{0} \not \perp d_{1}, d_{1} \not \perp d_{2}, \ldots, d_{m-1} \not \perp d, d_{0} \not \perp e_{1}, e_{1} \not \perp e_{2}, \ldots, e_{n-1} \not \perp d(m \neq n)$ in $\{R\}_{i}$, where $d_{0}, d_{1}, \ldots, d_{m-1}, d, e_{1}, \ldots, e_{n-1}$ are distinct labels. Furthermore, the degree is well-defined for all labels included in $\{R\}_{i}$ because any new label added in Step 1 or Step 2 is related to a label already included in $\{R\}_{i}$.

We define set $\Gamma(b)$ as $\Gamma(b)=\{A \mid b: A \in \Gamma\}$. Because the number of subformulas of $A$ is finite and the number of negations of propositional 
variables that appears in $A$ is also finite, $\left|\Gamma_{i}(b) \cup \Delta_{i}(b)\right|$ is also finite. Furthermore, from the same reasoning, there exists a smallest $k$ such that if $k \leq i$, then $\left|\Gamma_{i}(b) \cup \Delta_{i}(b)\right|=\left|\Gamma_{k}(b) \cup \Delta_{k}(b)\right|$. We say that $b$ is already maximal in $\{R\}_{i} \Gamma_{i} \Rightarrow \Delta_{i}$ if $i$ is larger than such $k$.

THEOREM 4.5. There is an effective procedure that determines whether formula $A$ is valid in $O$-models.

Proof: We show that we can stop the procedure to construct a canonical model for \{\}$\Rightarrow a: A$.

Suppose that the complexity of $A$ is $n$. If the degree of $b$ is greater than $n$ in $\{R\}_{i}$, and if $b: B$ is included in $\Gamma_{i}$ or $\Delta_{i}$, then $B$ is a propositional variable or the negation of a propositional variable because all procedures in Step 1 decrease the complexity of formulas, and Step 2 only creates the negation of propositional variables.

Suppose that the degree of $b$ is $n, \Gamma_{i}(b)=\left\{p_{1}, p_{2}, \ldots, p_{t}, \neg q_{1}, \neg q_{2}, \ldots, \neg q_{u}\right\}$, $\Delta_{i}(b)=\left\{r_{1}, r_{2}, \ldots, r_{v}, \neg s_{1}, \neg s_{2}, \ldots, \neg s_{w}\right\}$, and $b$ is already maximal. Note that all $q_{m}$ are included in $\left\{r_{1}, r_{2}, \ldots, r_{v}\right\}$ because $b$ is maximal. In Step 1, we have to add $b \not \perp c_{m}(1 \leq m \leq w)$ to $\{R\}_{i}$ for all $m$ and add $c_{m}: s_{m}(1 \leq$ $m \leq w)$ to $\Gamma_{i}$ for all $m$. In Step 2, we have to add $b \not L d_{m}(1 \leq m \leq v)$ to $\{R\}_{i}$ for all $m$ and add $d_{m}: \neg r_{m}(1 \leq m \leq v)$ to $\Gamma_{i}$ for all $m$. All $c_{m}$ and $d_{m}$ are new labels. Furthermore, in Step 1, we have to add $q_{m}(1 \leq m \leq u)$ to $\Delta_{i}$ labeled by $c_{m}(1 \leq m \leq w)$ and $d_{m}(1 \leq m \leq v)$, and we also have to add $d_{m}: r_{m}(1 \leq m \leq v)$ to $\Delta_{i}$. We denote this new sequent by $\{R\}_{j} \Gamma_{j} \Rightarrow \Delta_{j}$.

We prove that we can stop the process for labels $c_{m}$ and $d_{m}$. Although all $c_{m}: q_{m} \in \Delta_{j}$ and all $d_{m}: q_{m} \in \Delta_{j}$ request new labels in Step 2, we do not need new labels because label $b$ already has its role; that is, because $b: \neg q_{m} \in \Gamma_{j}$ and $b \not \perp c_{m} \in\{R\}_{j}$, and because of the procedure for making symmetric relations in Step 2, the existence of $b$ is sufficient to prove Lemma 4.1. Similarly, although $d_{m}: r_{m} \in \Delta_{j}$ requests a new label in Step 2, we do not need a new label because label $d_{m}$ already has its role.

Therefore, a label with a degree greater than $n+2$ is not needed. Furthermore, the number of labels related to a given label is finite because, in the above procedure, one labeled formula can create at most one relation under the conditions of the procedure. Therefore, we can stop the procedure from constructing a canonical model in a finite number of steps without any loss of information.

Although there are multiple possibilities that depend on the choice of Step 1 for $\wedge$ in $\Delta_{i}$ and $\rightarrow$ in $\Gamma_{i}$, because the number of formulas appearing in $a: A$ is finite, the number of procedures is also finite. 
If one of these finite procedures creates a canonical model, then we conclude that \{\}$\Rightarrow a: A$ is not provable and $A$ is not valid. If these finite procedures does not create a canonical model, then we conclude that \{\}$\Rightarrow a: A$ is provable and $A$ is valid.

For example, when we construct a canonical model for \{\}$\Rightarrow a:(q \rightarrow$ $p$ ), the procedure in this paper adds a number of $b_{n}: p$ in $\Delta_{i}$ (by Step 1), $b_{n}: \neg p$ in $\Gamma_{i}$ (by Step 2), and $b_{n-1} \not \perp b_{n}$ in $\{R\}_{i}$ after some steps. We can easily check that more than four labels are redundant.

$$
\{\} \Rightarrow a:(q \rightarrow p)
$$

$\downarrow$ Step 1

$$
\left\{a \not \perp b_{1}\right\} b_{1}: q \Rightarrow a:(q \rightarrow p), b_{1}: p
$$

\section{$\downarrow$ Step 2}

$\left\{a \not \perp a, b_{1} \not \perp b_{1}, b_{2} \not \perp b_{2}, a \not \perp b_{1}, b_{1} \not \perp a, b_{1} \not \perp b_{2}, b_{2} \not \perp b_{1}\right\} b_{2}: \neg p, b_{1}: q \Rightarrow$ $a:(q \rightarrow p), b_{1}: p$

$\downarrow$ Step 1 and Step 2 (Processing from here is superfluous).

$\left\{a \not \perp a, b_{1} \not \perp b_{1}, b_{2} \not \perp b_{2}, b_{3} \not \perp b_{3}, a \not \perp b_{1}, b_{1} \not \perp a, b_{1} \not \perp b_{2}, b_{2} \not \perp b_{1}, b_{2} \not \perp\right.$ $\left.b_{3}, b_{3} \not \perp b_{2}\right\} b_{3}: \neg p, b_{2}: \neg p, b_{1}: q \Rightarrow a:(q \rightarrow p), b_{1}: p, b_{2}: p$

THEOREM 4.6. LGOI is decidable. That is, there is an effective procedure that determines whether labeled sequent $\{R\} \Gamma \Rightarrow \Delta$ is provable in LGOI.

Proof: We can use almost the same method as that in the proof of Theorem 4.5; that is, we can stop the procedure from constructing a canonical model for $\{R\} \Gamma \Rightarrow \Delta$ in the same manner. We apply the notion of degree to all labels that appear in $\{R\}$. We say that the degree of label $b$ for $a \in\{R\}_{L}$ is $n(\geq 2)$ in $\{R\}_{i}$ if there exist labels $c_{1}, c_{2}, \ldots, c_{n-1}$ such that $a \not \perp c_{1}, c_{1} \not \perp c_{2}, \ldots, c_{n-1} \not \perp b$ are included in $\{R\}_{i}, a, b, c_{1}, c_{2}, \ldots, c_{n-1}$ are distinct labels, and $b, c_{1}, c_{2}, \ldots, c_{n-1}$ do not appear in $\{R\}$. We define the 
degree of $a \in\{R\}_{L}$ as 0 , and if $a \not L b \in\{R\}_{i}$ and $b \notin\{R\}_{L}$, then we define the degree of $b$ as 1 .

Because the number of formulas that appear in $\Gamma \cup \Delta$ is finite and the number of labels that appear in $\{R\}$ is also finite, we can use the same argument as that in the proof of Theorem 4.5. Although there is a possibility that there is a confluence or loop in $\{R\}$, because the procedure does not make a confluence or loop, and because of the definition of degree, we can confirm that the proof is not affected by these conditions.

If one of these finite procedures creates a canonical model of $\{R\} \Gamma \Rightarrow$ $\Delta$, then we conclude that $\{R\} \Gamma \Rightarrow \Delta$ is not provable. If these finite procedures do not create a canonical model of $\{R\} \Gamma \Rightarrow \Delta$, then we conclude that $\{R\} \Gamma \Rightarrow \Delta$ is provable.

\section{Acknowledgements}

I wish to thank R. Kashima and the anonymous reviewers for very helpful comments on earlier version of this paper. I also would like to thank R. Gore for making a proposal to start researching about labeled sequent calculi for quantum logic.

\section{References}

[1] M. L. D. Chiara and R. Giuntini, Quantum Logics, Handbook of Philosophical Logic 2nd Edition 6 (2001), pp. 129-228.

[2] C. Faggian and G. Sambin, From Basic Logic to Quantum Logics with Cut-Elimination, International Journal of Theoretical Physics 37(1) (1998), pp. 31-37.

[3] G. M. Hardegree, Material Implication in Orthomodular (and Boolean) Lattices, Notre Dame Journal of Formal Logic 22(2) (1981), pp. 163-182.

[4] Z. Hou, A. Tiu and R. Gore, A Labelled Sequent Calculus for BBI: Proof Theory and Proof Search, TABLEAUX 2013 (2013), pp. 172-187.

[5] S. Negri, Proof Analysis in Modal Logic, Journal of Philosophical Logic 34 (2005), pp. 507-544.

[6] S. Negri, Proof theory for modal logic, Philosophy Compass 6(8) (2011), pp. 523-538. 
[7] H. Nishimura, Sequential Method in Quantum Logic, The Journal of Symbolic Logic 45(2) (1980), pp. 339-352.

[8] H. Nishimura, Proof Theory for Minimal Quantum Logic I, International Journal of Theoretical Physics 33(1) (1994), pp. 103-113.

[9] H. Nishimura, Proof Theory for Minimal Quantum Logic II, International Journal of Theoretical Physics 33(7) (1994), pp. 1427-1443.

Tokyo Institute of Technology

School of Computing

Department of Mathematical and Computing Science

e-mail: kawano.t.af@m.titech.ac.jp 